\title{
Identifying the most important outcomes for systematic reviews of interventions for rhinosinusitis in adults: working with Patients, Public and Practitioners*
}

\author{
Claire Hopkins', Carl Philpott², Sally Crowe³, Sandra Regan³, \\ Aneeka Degun ${ }^{4}$, Iliatha Papachristou ${ }^{5}$, Anne G.M. Schilder ${ }^{4}$ \\ ' Guy's \& St Thomas' NHS Trust, King's College London, United Kingdom \\ 2 University East Anglia, Norwich and James Paget Hospital, Great Yarmouth, United Kingdom \\ ${ }_{3}^{3}$ Crowe Associates, Thame, United Kingdom \\ ${ }_{4}^{4}$ UCL Ear Institute, Royal National Throat, Nose and Ear Hospital, London, United Kingdom \\ Department of Applied Health Research, UCL, United Kingdom
}

Rhinology 54: 20-26, 2016

DOI:10.4193/Rhino15.199

*Received for publication:

July 21,2015

Accepted: August 27, 2015

\begin{abstract}
Introduction: Promoting the assessment of health interventions using outcomes that matter to patients and practitioners is a key principle of Cochrane. Cochrane UK therefore commissioned the OMIPP project: Outcomes that are Most Important for Patients, Public and Practitioners to identify the outcomes they felt most important and should be evaluated in Cochrane reviews of health interventions for Chronic Rhinosinusitis (CRS).
\end{abstract}

Methodology: Using direct emailing, social media and printed cards, an online survey was distributed to a wide range of people involved in the care of patients with CRS. Patients and practitioners were asked to list the 3 outcomes from treatments most important to them. Responses were analysed through development of a thematic framework based on the data.

Results: Two hundred and thirty-five people completed the survey; 155 practitioners and 80 patients. Respondents provided 653 suggestions of important outcomes. 73\% concerned symptoms of CRS, (nasal discharge or drip, facial pain, nasal blockage, headache, impaired sense of smell, congestion and breathing difficulties); $9 \%$ concerned quality of life, $4 \%$ reducing the need for further treatment and $4 \%$ side effects of treatment. Objective measurements of disease formed only $3 \%$ of responses. There was high level of agreement between patients and practitioners. Of 10 current Cochrane reviews on CRS, 9 include symptomatic outcomes identified by our survey as most important to patients and healthcare practitioners.

Conclusions: We have identified outcomes that both patients and their doctors consider should be included in reviews evaluating treatments of rhinosinusitis. We recommend that primary outcomes in future reviews focus on symptom-based outcomes. The ability to extract these data from relevant trials is dependent upon their inclusion in trials, and so it is important that building on this work a core outcome set for rhinosinusitis research is developed.

Key words: chronic rhinosinusitis, outcomes, outcomes research, endoscopic sinus surgery, effectiveness trials

\section{Introduction}

One of the principles of Cochrane is to promote the assessment of health questions using outcomes that matter to people making choices in health and health care, that is the people living with certain conditions and those making clinical decisions about their management. Cochrane authors and editors however face challenges to implement this principle when seeking to combine data from trials that often address a range of outcomes. In many instances the review teams select outcomes for their review based on their own views and experiences, rather 
Cochrane (http://www.cochrane.org) is an independent, global network working to promote evidence-informed health decision-making by producing high-quality, relevant and accessible systematic reviews. Cochrane Reviews investigate the effects of interventions for prevention, treatment, and rehabilitation. They also assess the accuracy of a diagnostic test for a given condition in a specific patient group and setting. Cochrane Reviews follow a clearly defined methodology to systematically identify, appraise and where possible combine the results of randomised controlled trials. There are currently over 5,400 Cochrane Reviews published online in the Cochrane Library.

Cochrane UK (http://uk.cochrane.org) is one of the more than 40 regional centres supporting the global work of Cochrane and maximizing the use and impact of Cochrane Reviews for the UK and Ireland. Cochrane UK is funded by the National Institute for Health Research (NIHR) and hosted by the Oxford University Hospitals NHS Trust.

Cochrane ENT (http://ent.cochrane.org) is based at Cochrane UK and works collaboratively with contributors around the world to produce systematic reviews on all aspects of ear, nose and throat (ENT) disorders and head and neck cancers.

than those of a wider group of people with relevant perspectives.

In 2014 Cochrane UK therefore commissioned the OMIPP project: Outcomes that are Most Important for Patients, Public and Practitioners. The pilot project aimed to identify the best method to develop therapeutic outcomes for Cochrane Reviews that are important for patients, public and health practitioners using the James Lind Alliance (http://www.lindalliance.org) experience of working with these groups. The pilot tested three different approaches across three different conditions: 1) secondary analysis of the existing qualitative literature (including a module of Health Talk Online) to identify important outcomes in breastfeeding; 2) a face-to-face workshop around outcomes in asthma and 3) an online survey to identify important outcomes in rhinosinusitis. This paper focuses on the outcomes in this last condition. The results of the first two and a comparison of the different approaches used in the pilot will be presented elsewhere.

Cochrane ENT chose chronic rhinosinusitis as a priority for their pilot project because of its large health burden to patients and the NHS. An estimated $10 \%$ of the UK adult population are affected by chronic rhinosinusitis ${ }^{(1)}$ and some studies have shown a greater impact on quality of life than heart disease and back pain ${ }^{(2)}$. Each year more than 600,000 adults are treated for CRS by their GPs and $91 \%$ receive antibiotics, often with repeated courses ${ }^{(3)}$. Over 120,000 patients with rhinosinusitis attend hospital each year, with around 40,000 subsequently undergoing sinus surgery ${ }^{(3)}$. There has been little research to assess what outcomes should be measured for this condition, especially from the patients' perspective. Individual trials have mostly relied on objective measures, which include sinus ostial patency, endoscopic or radiological disease severity, tests of nasal airflow and sense of smell. Some have included patient reported outcomes like ratings of overall and individual symptom severity ${ }^{(4)}$. The wide number of different outcomes used across trials limits meta-analysis and thereby the impact of the reviews. Cochrane ENT saw the OMIPP pilot project as an important step towards developing a core set of outcomes to be used across their reviews of medical and surgical treatments for chronic rhinosinusitis in adults.

\section{Methodology}

\section{Study design}

As allocated by the OMIPP project, this pilot used an online survey to gather the outcomes for Cochrane Reviews in chronic rhinosinusitis that are important for patients, public and practitioners. The survey was developed following a review of the literature on outcomes used in chronic rhinosinusitis, and informed by the team's experience with developing similar surveys for James Lind Alliance prioritisation projects. It was designed to achieve two main goals; 1 ) to gather outcomes of chronic rhinosinusitis treatments of interest to both health practitioners and people with the condition, and 2) to identify the most important of these. The survey was tested in a small group of practitioners and patients and modified to minimise respondent burden and to ensure acceptability to both.

The cover page of the survey explained the context of the pilot and asked participants to consider the outcomes of treatment for rhinosinusitis that mattered to them and list their top 3 in a free text format. There were no predefined codes or prompts given to guide responses. Since we thought that patients might be unfamiliar with the terms acute and chronic, we referred to 'rhinosinusitis'.

The exact questions were as follows:

1. For people with rhinosinusitis: What results from treatments are most important to you?

2. For health professionals: What are the most important outcomes that you want from treatments for people with rhinosinusitis?

We targeted respondents so that outcomes were expected to focus around chronic rather than acute rhinosinusitis. We used Survey Monkey software to host the survey; online sur- 
Table 1. Sub-themes: Symptoms rated as important as treatment outcomes in rhinosinusitis by practitioners and patients.

\begin{tabular}{|c|c|}
\hline Sinonasal symptoms & Other symptoms \\
\hline Nasal discharge/drip & $\begin{array}{l}\text { "improve, reduce, resolve" } \\
\text { symptoms }\end{array}$ \\
\hline Nasal obstruction/blockage & Sleep \\
\hline Headache & Fatigue \\
\hline Smell & $\begin{array}{l}\text { Specific Patient Reported Out- } \\
\text { come Measures (PROMs) such as } \\
\text { the SNOT-22 (includes symptom } \\
\text { evaluation) }\end{array}$ \\
\hline Congestion & $\begin{array}{l}\text { "improve, reduce, resolve" } \\
\text { symptoms }\end{array}$ \\
\hline Pain & \\
\hline
\end{tabular}

veys have proven popular and (cost-) effective in other related initiatives such as the James Lind Alliance. Social Media (Facebook, Twitter) and existing email networks (ENT-UK, the British Rhinological Society and British Society of Asthma and Clinical Immunology, research and clinical colleagues) were used to reach patients with the condition and health-care professionals. Information was sent to members of Fifth Sense, a charity for people affected by smell and taste disorders, some of whom have CRS. A flyer with a link to the survey was developed and circulated to clinicians to distribute their patients.

An incentive was offered for people completing the survey; respondents were entered into a draw for $5 £ 25$ online vouchers. The survey was open from the 19th December 2014 until the 10th February 2015.

\section{Data analysis}

All free text data was downloaded from Survey Monkey into an Excel spreadsheet, with a unique allocated ID for each responder and outcome. Responses were kept within their professional and patient categories to allow comparison between the two data sets.

Two team members analysed the data in two stages; firstly applying thematic analysis that allowed for developing themes from the data itself. All submissions were read to identify themes with discussion with the expert group before the final themes were selected. Secondly, each submission was read again and allocated to one or more themes. With input from the expert team, those that were considered to be out of scope were removed. An outcome was considered to be a result or consequence that could occur as a result of an intervention; this could include both beneficial outcomes and adverse events. Many of the responses considered out of scope related to suggestions for new or improved interventions (ie topics for future

\section{E Health professionals $\quad$ People with rhinosinusitis}

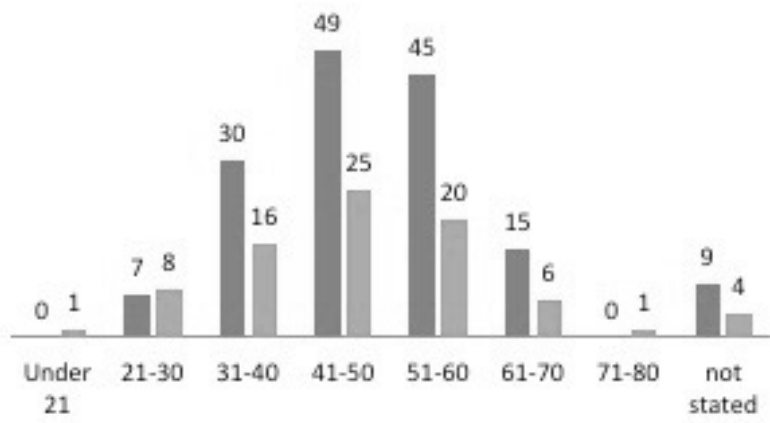

Figure 1. Age of respondents. research).

Finally, the Cochrane ENT Disorders Group provided a list of the primary and secondary outcomes that had been used in systematic reviews for interventions for chronic rhinosinusitis and we mapped the survey themes to that list, noting the prevalence of response.

\section{Results}

\section{Overview of responses}

There were over 900 responses to the survey; approximately 600 of these were identified as spam and were removed. We extracted 235 usable responses, from 155 practitioners and 80 people with rhinosinusitis. From the 235 usable responses there were 653 suggestions of important outcomes. Of these 549 (169 from people with rhinosinusitis, and 380 from practitioners) fitted our description of an outcome. Items that were removed included suggestions for treatments, comments on the properties of different outcomes tools, and items that we were unable to understand ; this totalled to $104 ; 56$ from professionals and 48 from patients. We contacted one respondent where we needed further clarification.

More male practitioners responded to the survey than female (109 and 18 respectively), which is likely to reflect the gender balance in the specialty of Otorhinolaryngology in the UK. In people with rhinosinusitis more women than men responded (59 and 39 respectively). Age ranges reflected those of practitioners and patients with CRS (Figure 1). Of the 155 practitioners who responded, 116 were ENT surgeons 11 respiratory and allergy specialists, 8 GPs and nurses $(n=3)$.

Patients with rhinosinusitis were asked to self-classify according to their understanding of their condition (acute, recurrent acute, 


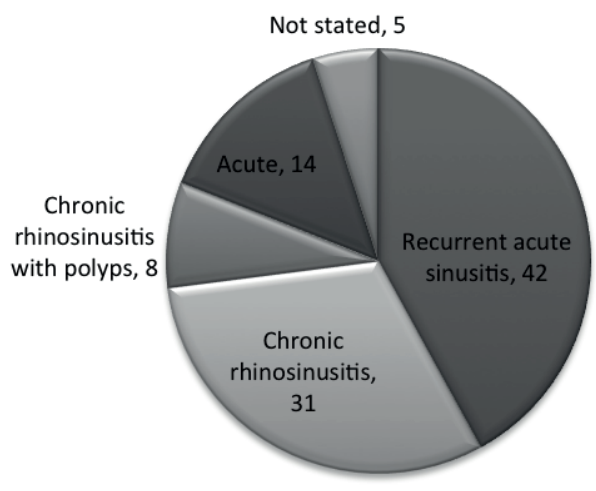

Figure 2. Patient rated categorisation of sinusitis status, $\%$.

chronic, chronic with polyps) (Figure 2). A total of $52 \%$ of CRS respondents reported on-going problems at time of survey completion.

\section{Themes in the data}

The first analysis of the data resulted in 11 themes, which after expert team discussion was reduced to eight themes:

1. Improvement of symptoms

2. Reducing need for surgery

3. Quality of Life

4. Patient acceptability of medication

5. Adverse and side effects of treatments

6. Objective outcome measures

7. Other respiratory conditions (including Asthma)

8. Reducing acute exacerbations and resulting consultations

Figure 3 shows the spread of responses across themes: most responses from both practitioners and people living with rhinosinusitis referred to 'improvement of symptoms'. Eighty percent of patients and $69 \%$ of practitioners prioritised symptom outcomes.

Focussing further on theme 1, Table 1 summarises its subthemes within two main areas; sinonasal symptoms and other symptoms. Some respondents chose to put either "improve, reduce, resolve" symptoms as one of their choices (mostly practitioner) and as these did not define specific symptoms these were assigned to the 'other' category

In terms of individual symptoms (Figure 4), nasal discharge (drip) and nasal obstruction and blockage were the most frequent outcomes suggested by our respondents.

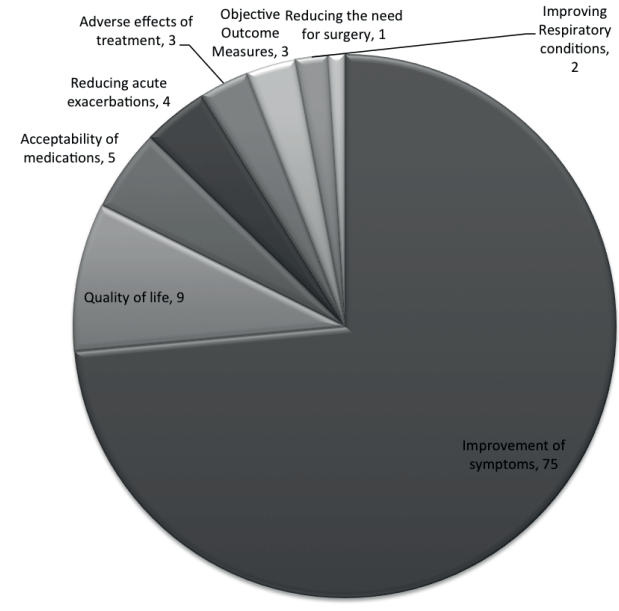

Figure 3. Outcomes groups under main themes, \%.

\section{Comparison of survey outcomes to those used in Cochrane Reviews \\ Of the current chronic rhinosinusitis reviews, 9 include sympto- matic improvement as an outcome, with this being used as the primary outcome in reviews published since 2011. Only three outcomes included in the reviews did not map onto a survey theme: changes in the microbiome, treatment drop-outs and global changes in health status. \\ We found good alignment between the survey results and cur- rent outcome measures included in systematic reviews. Our re- sults strengthened a recommendation by the Cochrane Review Group to use symptom improvement as the primary outcomes in all CRS reviews. Global quality of life, adverse effects of treat- ment, impact on respiratory diseases, reducing need for future treatment (both medical and surgical) should be considered as secondary outcomes.}

\section{What were the most effective ways of publicising the sur- vey?}

We asked survey respondents to tell us how they came to the survey; 217 answered. Excluding ENT specialists, we estimate that the twitter activity was most effective in driving people to the survey. It also helped us use existing patient and public involvement opinion formers and influencers to share the survey. Twitter accounted for 44 people completing the survey. We had 7 people register for the survey via the INVOLVE People in Research website (which matches the publics' interest in research to projects) and also the Cochrane Consumer Network and Facebook page (7 people). The most effective routes to engage ENT specialists were peer-to-peer contact and personal endorsement from the clinicians in the working group, and contact lists for ENT professional organisations $(n=74)$. Fifty two people described their route to the survey via 'email' and it is likely that some of these will fit in the peer to peer, or mailing list category. 


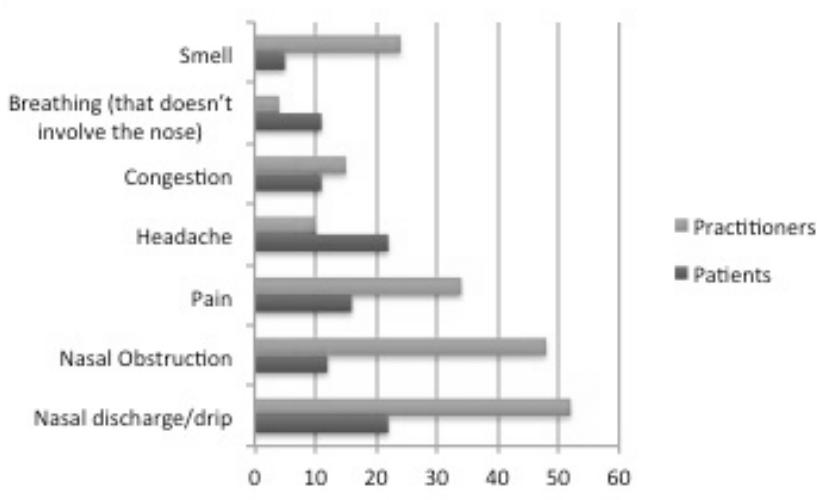

Figure 4. Individual symptoms rated as important by practitioners and patients.

The team designed and printed Postcards for the survey and offered these to practitioners to give to patients in clinics. Only 1 respondent cited this as their route to the survey.

\section{Discussion}

Improvement of symptoms was considered the most important outcome that should be included in Cochrane Reviews evaluating interventions for RS, by both patients and health care providers. While some listed individual symptoms, others described symptoms in general or the use of questionnaires such as the SNOT-22 ${ }^{(5)}$, which capture many symptoms that are reported by patients with CRS. Of note, the 4 most commonly named symptoms (nasal obstruction, nasal discharge, facial pain and loss of sense of smell) are those included as the diagnostic criteria for rhinosinusitis, defined by the European Position Paper on Rhinosinusitis and Nasal Polyps. These are also captured by the SNOT-22 and RSOM-31(6) patient-rated outcome instruments. One exception is headache; some patients listed headache specifically, while others reported facial pain. However, when the RSOM-31 underwent item deletion as part of its psychometric development, headache was removed because of redundancy with facial pain, and does not appear in the SNOT22. Further work is perhaps required to confirm that these do not need to be measured independently in order to capture the full impact of CRS on the patient. 'Quality of life' was the second most important theme; health related quality of life is also captured by these disease-specific instruments. Our results suggest that reviews should consider evaluation of symptoms as their primary outcome.

We found good alignment between the survey results and cur-

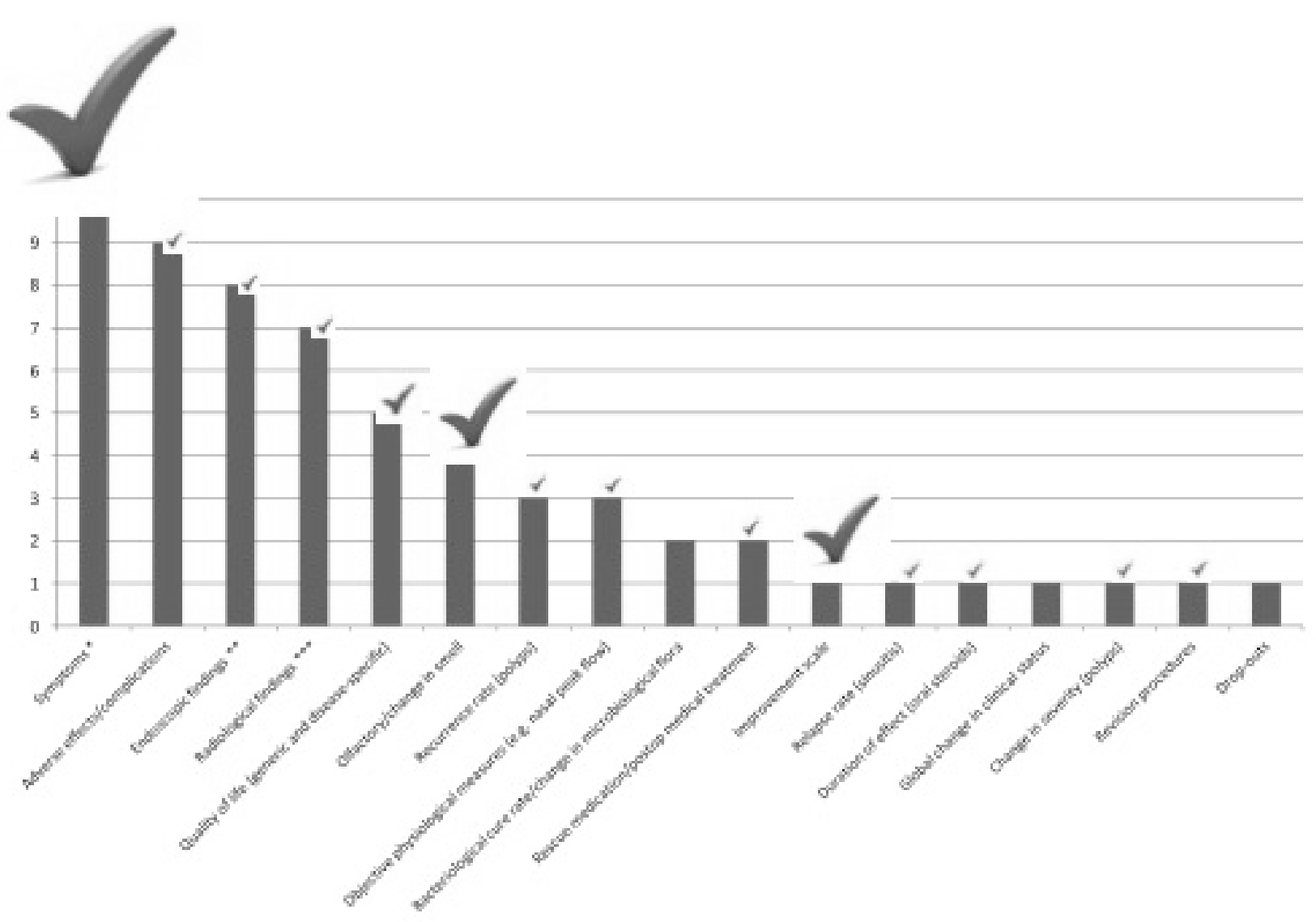

Figure 5. Outcomes used in Cochrane Reviews of interventions for CRS, with frequency of given outcome in survey denoted by size of the tick. 
rent outcome measures included in systematic reviews. Our results strengthened a recommendation by the Cochrane Review Group to use symptom improvement as the primary outcomes in all CRS reviews. Global quality of life, adverse effects of treatment, impact on respiratory diseases, reducing need for future treatment (both medical and surgical) should be considered as secondary.

Objective outcomes formed only $3 \%$ of responses, reflecting and increased awareness of the importance of outcomes that reflect the burden of disease from the patient's perspective and reduced reliance on clinician rated or 'objective' outcomes. Of course, one limitation of this study as that this may simply reflect a lack of patient awareness of objective outcomes, although ENT surgeons also rated them as having low importance.

There have been numerous studies that have compared and often found only a weak correlation between objective outcome measures, such as polyp grading ${ }^{(7)}$, olfactory testing ${ }^{(8)}$ or objective tests of nasal airflow ${ }^{(9)}$, while other studies have found good alignment between patient rated outcomes and objective measures such as healthcare utilisation ${ }^{(10,11)}$. Objective measures evaluate a different aspect of disease and still play an important role in outcome assessment, and we believe they should be included in secondary measures, alongside the other themes identified. The high rating of patient rated outcomes by clinicians was reassuring, demonstrating a good connection with their patients' perspectives. Recent National Institute of Healthcare Research feedback on a trial proposal submitted by the team questioned the choice of a patient rated primary outcome as the primary outcome and suggested that an objective measure would be preferable (personal communication). There remains some reluctance to the use of patient rated symptom based outcomes, but this project lends further support to their use. Interestingly, changes in the microbiome were not considered as an important outcome by either patients or practitioners. However, with increasing antibiotic resistance, this will remain an important consideration in trials of antibiotic effectiveness in rhinosinusitis and perhaps is something that all clinicians need to be more considerate of.

One limitation of this project was that we sought to evaluate outcomes for reviews of interventions in rhinosinusitis as a whole group. While the Cochrane ENT group are primarily concerned with reviews of CRS, we did not specify this in the questionnaire, as we were unsure that patients would be able to accurately self-classify. It is possible that we would have received different responses if we had targeted only outcomes for CRS, however there was no significant difference in the responses given by patients self classifying as acute or chronic rhinosinusitis. We were not convinced that the patients who participated in the survey were able to accurately self-categorise their sinusitis according to robust definitions of acute, chronic and recurrent acute sinusitis. Firstly, there were far more patients self-categorising as recurrent acute sinusitis (RARS) than we would expect, as the estimated prevalence of RARS is $0.035 \%{ }^{(3)}$. Moreover, the majority of these patients reported that they had symptoms at the time of completing the survey, suggesting that they probably had CRS with acute exacerbations, rather than true RARS. We were also surprised that there seemed to be little differences in responses given by each of the subgroups. Due to this uncertainty we have not formally reported any subgroup analysis separating acute, RARS and chronic RS.

We removed nearly 600 spam responses, where nonsensical or no outcomes were reported that we believe were motivated by the prize draw. We retained only plausible outcomes, and we think it unlikely that any of the patient responses retained were fraudulently entered; indeed many of the respondents also made free text comments on living with sinusitis.

One of the aims of his project, commissioned by Cochrane UK, was to identify what outcomes are thought to be important to be included in systematic reviews. When comparing the outcomes identified by our survey, and those used in current Cochrane reviews of treatments in chronic rhinosinusitis, there is a high degree of alignment. In particular, there has been a shift in outcomes used in reviews away from ostial patency and other objective measures, towards patient rated symptom improvement. Indeed all reviews published since 2011 have included symptomatic improvement as the primary outcome. However, these outcomes can only form the basis of systematic review if they are included in the trials that they evaluate.

We plan to develop this project further to develop Core Outcome Measures for trials of interventions for ENT. The Core Outcome Measures in Effectiveness Trials (COMET) initiative has a clearly defined methodology to produce Core Outcome Sets and a role in improving Cochrane Reviews ${ }^{(12)}$. This process utilises two to four steps in refining responses collected from Stakeholders and achieve consensus through a Delphi technique ${ }^{(13)}$. One advantage to this methodology is that all possible outcomes are long-listed, and then sequentially ranked, which overcomes any lack of awareness of outcomes from either the patient or practitioners' perspective. In contrast, our pilot utilised only a single round and limited responses to the 3 considered most important. This forced choice ensured that people focused on the most important issues for them in one hit, and very few survey responders tried to fit in more than 3. Outcomes considered less important and not included may well be captured by early iterations of the Delphi process but are likely to fall out of subsequent iterations. We propose that our 'one- 
hit' method may be a more time and cost-effective means to achieve the same outcome; however comparison with a Delphi process addressing the same topic is required to make strong conclusions.

This pilot data has provided useful data on how best to engage with patients and healthcare providers, utilising social medial and established networks. It is essential that the views of patients and those that manage CRS in all settings are considered when developing this project further.

\section{Conclusion}

We have found that both people with CRS and those that treat them consider (improvement of) symptoms of the disease the most important outcomes that should be included in Cochrane Reviews of interventions for rhinosinusitis. We recommend that outcomes of future reviews focus on instruments that capture such symptoms and health related quality of life. Other important outcomes as prioritised by patients and practitioners are adverse effects and acceptability of treatments, impact on respiratory disease, avoiding the need for surgical intervention or ongoing health care utilisation through medication use and doctor visits.

We recommend that building on this work a core outcome set for chronic rhinosinusitis for use in future trials is developed.

\section{Acknowledgement}

We are grateful to Jenny Bellorini and Sam Faulkner of the Cochrane ENT Group for supporting the project. We also extend our thanks to Jim Boardman and Abi Dennington Price for their valuable comments from a patient perspective.

\section{Authorship contribution}

All authors contributed to all stages of the study and manuscript preparation.

\section{Conflict of interest}

None

\section{References}

1. Hastan D, Fokkens WJ, Bachert C, Newson RB, Bislimovska J, Bockelbrink A, et al. Chronic rhinosinusitis in Europe--an underestimated disease. A GA(2)LEN study. Allergy. 2011 Sep;66(9):1216-23.

2. Gliklich RE, Metson R. Effect of sinus surgery on quality of life. Otolaryngol Head Neck Surg. 1997 Jul;117(1):12-7.

3. Hopkins $\mathrm{CHC}$, Philpott $\mathrm{C}$. The care pathway if patients with chronic rhinsinusitis in the UK. British Academic Conference of Otolaryngology; Liverpool2015.

4. Fokkens WJ, Lund VJ, Mullol J, Bachert C, Alobid I, Baroody F, et al. European Position Paper on Rhinosinusitis and Nasal Polyps 2012. Rhinol Suppl. $2012 \operatorname{Mar}(23): 3$ p preceding table of contents, 1-298.

5. Hopkins C, Gillett S, Slack R, Lund VJ Browne JP. Psychometric validity of the 22-item Sinonasal Outcome Test. Clin Otolaryngol. 2009 Oct;34(5):447-54.

6. Piccirillo JF, Edwards D, Haiduk A, Yonan C Thawley SE. Psychometric and clinimetric validity of the 31-item rhinosinusitis outcome measure (RSOM-31). Am J Rhinol. 1995 Nov-Dec;9(6):297-306.

7. Aboud SK, Husain S, Gendeh BS. Comparison between endonasal endo- scopic polyp size scores and quality of life outcome after optimal medical treatment. Rhinology. 2014 Dec;52(4):334-40.

8. Minwegen F, Thomas JP, Bernal-Sprekelsen M, Dazert S, Minovi A. Predictive value of disease severity on self-reported rating and quantitative measures of olfactory function outcomes after primary endoscopic sinus surgery. A prospective study. Rhinology. 2014 Dec;52(4):437-43.

9. Akduman D, Yanilmaz M, Haksever M, Doner F, Sayar Z. Patients` evaluation for the surgical management of nasal obstruction. Rhinology. 2013 Dec;51(4):361-7.

10. Hopkins C, Andrews P, Holy CE. Does time to endoscopic sinus surgery impact outcomes in chronic rhinosinusitis? Retrospective analysis using the UK clinical practice research data. Rhinology. 2015 Mar:53(1):18-24

11. Hopkins C, Rimmer J, Lund VJ. Does time to endoscopic sinus surgery impact outcomes in Chronic Rhinosinusitis? Prospective findings from the National Comparative Audit of Surgery for Nasal Polyposis and Chronic Rhinosinusitis. Rhinology. 2015 Mar;53(1):10-7.

12. Prinsen $C A$, Vohra $S$, Rose MR, King-Jones S, Ishaque S, Bhaloo Z, et al. Core Outcome
Measures in Effectiveness Trials (COMET) initiative: protocol for an international Delphi study to achieve consensus on how to select outcome measurement instruments for outcomes included in a 'core outcome set'. Trials. 2014;15:247.

13. Williamson P, Clarke M. The COMET (Core Outcome Measures in Effectiveness Trials) Initiative: Its Role in Improving Cochrane Reviews. Cochrane Database Syst Rev. 2012;5:ED000041.

Claire Hopkins

Guy's Hospital

Great Maze Pond

London SE1 9RT

United Kingdom

Tel: +44-201-71882215

E-mail: claire.hopkins@gstt.nhs.uk 